\title{
Prevenir y persuadir. La radio y las Visitadoras de Higiene en las décadas de 1930 y 1940 en la ciudad de La Plata
}

\section{Prevent and persuade. Radio and Social Workers of Hygiene in the 1930s and 1940s in La Plata city}

\author{
Canela Constanza Gavrila \\ Centro de Estudios de Trabajo Social y Sociedad \\ Universidad Nacional de La Plata \\ elcorreodecanela@gmail.com \\ (Argentina)
}

El propósito del siguiente artículo es analizar las acciones de persuasión y prevención que ejercieron las visitadoras de higiene social en las estaciones de radio de la ciudad de La Plata, en las que pusieron en tensión los roles atribuidos al género femenino, como también los deberes establecidos para su desempeño profesional. Esta preocupación se inscribe en dos intereses más amplios, por un lado, recuperar y repensar la historia del trabajo social comprendiendo a las visitadoras más allá de las prescripciones de los médicos y de los roles atribuidos a su género. Por otra parte, se pretende ponderar un relato de orden local que aporte nuevas miradas a la historia del trabajo social.

El trabajo se compone de dos apartados. En el primero se presentan algunas características de la Escuela de Visitadoras de Higiene de la Universidad Nacional de La Plata (UNLP), y en el segundo apartado se atiende a la participación de las visitadoras como conferencistas radiales.

Palabras Clave: Visitadoras de higiene - Persuadir - Radio 


\begin{abstract}
The purpose of this article is to analyze the persuasive and preventing actions that social workers carried out in the radial stations of La Plata city. These actions put pressure on gender roles and the established duties of their professional performance. It is based on two wider interests: first of all, to recover and rethink the social working history understanding social workers beyond medical prescriptions and gender roles. Secondly, this article considers the relevance of local narrations to generate other points of view about women participation in the history of social working.

The first part of the article introduces the characteristics of the School of Social Workers of Hygiene of the University of La Plata, and in the second section, it is focused on the participation of the Social Workers of Hygiene as radial conference speakers.
\end{abstract}

Key Words: Social workers - Persuade - Radio

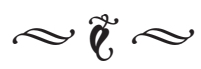

\section{Introducción}

La participación de las mujeres en las tareas de asistencia resulta de una asociación histórica que vincula a las féminas con las tareas de caridad, beneficencia y auxilio de la comunidad. Desde fines del siglo XIX y durante las primeras décadas del siglo XX, la condición femenina ligada al amor, al altruismo y la buena voluntad era contrapuesta a la situación de las mujeres obreras que desafiaron la moralidad femenina y su condición maternal. ${ }^{1}$ La temprana expansión de la educación primaria en el país como parte del proyecto modernizante convocó a la participación de mujeres de los sectores medios para una capacitación técnica, científica y administrativa que les otorgó reconocimiento social y también una cuota

1. LOBATO, Mirta Zaida, Historia de las trabajadoras en la Argentina (1869-1960), Edhasa, Buenos Aires, 2007, p. 66. de poder que las distinguió de los sectores populares a quienes pedagogizaba. ${ }^{2}$ Así, actividades como la asistencia permitieron a las jóvenes de sectores sociales medios y ascendentes una formación profesional capaz de conciliar los "deberes femeninos" y maternales atribuidos en la época con el mundo laboral.

La Escuela para Visitadoras de Higiene Social (en adelante EVHS) se inscribe en esta línea y constituye el primer antecedente universitario de la actual profesión de Trabajo Social. Esta institución permitió a las mujeres tender puentes entre aquellos conocimientos privativos del orden doméstico (como la limpieza de la casa, la ventilación, hasta

2. DI LISCIA, Maria Silvia, "Renovación de la historia sobre instituciones, profesionales y salud" en BIERNAT, Carolina, RAMACCIOTTI, Karina (editoras) Historia de la salud y la enfermedad. Bajo la lupa de las ciencias sociales, Biblos, Buenos Aires, 2014, pp. 123- 124. 
consejos para el amantamiento, entre otros) junto con aquellos producidos por los médicos higienistas a cargo de la escuela. Mediante acciones de profilaxis, prevención social y educación sanitaria buscaron luchar contra las costumbres perniciosas para el individuo y su comunidad.

Particularmente, las profesiones auxiliares a la medicina lidiaron con un campo tempranamente profesionalizado $y$ jerarquizado, donde las mujeres batallaron por los espacios de poder. Las áreas auxiliares como la enfermería, las visitas de higiene, la obstetriciay la puericultura, fueron profesiones que pretendieron alejar a las féminas de la competencia del monopolio masculino de los saberes médicos y vincularlas con actividades ligadas al cuidado y a la relación con otras mujeres. $^{3}$

Algunos estudios consideran que la convocatoria exclusiva a mujeres en la EVHS facilitó la intervención del Estado, puesto que las "legitimadas por su género" podían ingresar en la vida doméstica de los pobres urbanos a fin de modificar sus pautas de conducta desde un carácter doctrinario y moralizador capaz de encuadrar a los sectores peligrosos en las nuevas relaciones de capital- trabajo de principios del siglo XX. Estos argumentos se valen de considerar a las visitadoras como agentes encargadas de entablar relaciones amistosas y familiares con los sectores populares con el objetivo

3. NARI, Marcela, Politicas de Maternidad y Maternalismo Politico, Biblos, Buenos Aires, 2004, pp. 101-121; MARTIN, Ana Laura, "Mujeres y enfermería. Un asociación temprana y estable, 1886- 1940" en BIERNAT, Carolina, CERDÁ, Juan Manuel, RAMACCIOTTI, Karina (directores) La salud pública y la enfermería en Argentina, Universidad Nacional de Quilmes, Bernal, 2015, pp. 257-258. de facilitar la modificación de patrones de conducta y prácticas cotidianas en los hogares. ${ }^{4}$ Si bien es innegable que el Estado tomó los saberes aprehendidos en la vida doméstica de las mujeres, los jerarquizó a través de títulos profesionales, e incluso tomó prenda de ello para legitimar su intervención; estos trabajos centralizan su mirada sobre el carácter afectivo, amoroso, conservador y moralizante con que las visitadoras realizaban sus labores de prevención y profilaxis, y dejan de lado otras acciones disruptivas/creativas de estas nuevas profesionales, como la toma de la palabra en medios de comunicación radiales, en instituciones y en espacios laborales, e incluso, olvidan las estrategias persuasivas con que las visitadoras irrumpieron en el espacio público para convencer a la población de acercarse a los dispensarios de salud, y también para exhortar a profesionales y gobernantes acerca de su compromiso con la sanidad de madres, niños y de la sociedad en su conjunto. Por otra parte, estos estudios han focalizado su atención en la ciudad de Buenos Aires, por lo que aíslan otras experiencias que pueden aportar nuevas miradas para la comprensión de las acciones realizadas por las mujeres en el desarrollo de la profesión.

Las primeras agentes del servicio social de la ciudad de La Plata no sólo intervinieron en la vida cotidiana de los sectores populares mediante labores en dispensarios o en visitas domiciliarias, incluso se valieron de una estrategia novedosa para el ingreso

4. Ver por ejemplo: GRASSI, Estela, La mujer y la profesión de asistente social: el control de la vida cotidiana, Humanitas, Buenos Aires, 1989, pp. 159- 161; OLIVA, Andrea, Trabajo social y lucha de clases, Imago mundi, Buenos Aires, 2007, pp. 69-70; PARRA, Gustavo, Antimodernidad y Trabajo Social. Orígenes y expansión del Trabajo Social Argentino, Espacio Editorial, Buenos Aires, 2007, pp. 65-70. 
en la cotidianeidad: la participación en radioconferencias. La intervención en el joven espacio radial platense permitió una nueva construcción de la relación público-privado donde las mujeres que allí se enunciaban comenzaron a resignificar las relaciones de género desde el ingreso en el paisaje sonoro local, donde antes no eran audibles masivamente. ${ }^{5}$

La característica singular de las radioconferencias de las visitadoras fue la persuasión, es decir, el uso de argumentos y razones para convencer al público de modificar sus conductas habituales, e incluso de reformar las políticas sanitarias.

Desde fines de la década de 1930 e inicios de la década de 1940 estas profesionales disertaron en ciclos de propaganda higiénica organizados desde la Cátedra de Higiene y Medicina Preventiva de la Facultad de Ciencias Médicas (en adelante FCM) emitidas por Radio Universidad LR11 como parte de las actividades de extensión que realizaba la Universidad Nacional de La Plata (en adelante UNLP). También lo hicieron desde las radioconferencias organizadas por la Liga Popular contra la Tuberculosis de la Provincia de Buenos Aires (LPTPBA) en Radio Provincia LS11, que desde su creación por el Gobernador Manuel Fresco en 1937 tuvo por objetivo divulgar obras y discursos de gobierno que alentaran a la propagación de sus lemas de "orden, disciplina y jerarquía". ${ }^{6}$

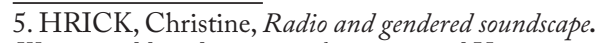
Women and broadcasting in Argentina and Uruguay, 1930- 1950, Cambridge University Press, Cambridge, 2015.

6. REITANO, Emir, Manuel Fresco. Entre la renovación 96
Este artículo tiene como objetivo analizar las acciones de persuasión y prevención que ejercieron las visitadoras de higiene social en los medios radiales de la ciudad de $\mathrm{La}$ Plata en los que estas jóvenes pertenecientes a sectores medios ascendentes de la sociedad tensionaron los roles maternales y amorosos establecidos para las mujeres que se desempeñaban como auxiliares del ámbito sanitario. Esta preocupación se inscribe en dos intereses más amplios, en principio, recuperar y repensar la historia de los inicios del trabajo social comprendiendo a las visitadoras más allá de las prescripciones de los médicos y de los roles atribuidos a su género con el objetivo de restituir un lugar de relevancia al interior de la historia profesional y en la historia de las mujeres. Por otra parte, pretendemos ponderar un relato de orden local de las funciones y conocimientos de las visitadoras.

\section{Auxiliares de la medicina preventiva. La Escuela para Visitadoras de Higiene Social de la ciudad de La Plata}

La ciudad de La Plata, capital de la provincia de Buenos Aires, se distinguía de otras ciudades del Estado nacional por estar proyectada desde sus orígenes como parte de la ciencia positivista, característica evidente en su planificación urbanística. El crecimiento urbano y poblacional, hacían temer por el despliegue de la cuestión social y con ella la expansión de las epidemias, hechos que permitieron a la higiene acoplarse con el proceso institucional suministrando un corpus normativo de orden prescriptivo

y el fraude. Archivo Histórico de la Provincia de Buenos Aires, La Plata, 2005, pp.29- 30. 
y proscriptivo al Estado, haciendo de los médicos actores centrales en la proyección de las políticas sociales. ${ }^{7}$

Aún así, y desde un lugar auxiliar, las mujeres se encontraron presentes en la atención socio-sanitaria a partir de la formación del Estado nacional, desde 1887 a través de su participación en la Sociedad de Beneficencia y en la Asistencia Pública de la ciudad. ${ }^{8}$

Sin embargo, desde las transformaciones producidas por la creciente industrialización desplegada desde el centenario hacia la década del treinta, los sectores gobernantes exigieron garantizar cuerpos saludables dotados de nuevos hábitos de comportamiento, por ello propiciaron la creación de instituciones sanitarias que garantizaran la salubridad de la clase obrera, ejemplo de ello fue el alcance de la cruzada por la lucha contra la tuberculosis. ${ }^{9}$ En la ciudad de La Plata, se inauguró el primer dispensario para el año 1922, y se replicó esta experiencia por intermedio de las acciones llevadas por la Cruzada Nacional contra la Tuberculosis. ${ }^{10}$ Para ello exigieron nuevos agentes que intervinieran sobre las problemáticas sociales desprendidas de las transformaciones económicas, es

7. VALLEJO, Gustavo, Escenarios de la cultura cientifica argentina. Ciudad y universidad (1882-1955),

CSIC, Madrid, 2007, pp. 53- 57.

8. GRAU, Carlos, La Sanidad en las ciudades y pueblos de la provincia de Buenos Aires, Dirección de Impresiones Oficiales, La Plata, 1954, pp. 74- 79.

9. ARMUS, Diego, La ciudad impura. Salud, tuberculosis y cultura en Buenos Aires, 1870-1950, Edhasa, Buenos Aires, 2007

10. ARRUA, Néstor, "Médicos higienistas y visitadoras frente a la tuberculosis en la ciudad de La Plata (19351943)", Revista Cátedra Paralela, Rosario, 2014, № 11, pp. 107- 134. decir: el desempleo, el crecimiento de las enfermedades sociales, el desarrollo de las epidemias, la problemática del alcoholismo, y además, buscaron garantizar la reproducción de la población para el acrecentamiento de la mano de obra.

En el año 1924 el Instituto de Higiene y la Cátedra de Higiene de la Facultad de Ciencias Médicas Universidad Nacional de Buenos Aires (en adelante UBA) crearon la Escuela para Visitadoras de Higiene Social, y catorce años más tarde el Dr. Pilades Dezeo replicó dicha experiencia en la Facultad de Ciencias Médicas (en adelante FCM) de la UNLP. ${ }^{11}$ En el discurso inaugural destacó que la amplitud del campo de la medicina preventiva no podía ser colmado por los esfuerzos de los médicos, y por ello, su carácter social reclamaba la incorporación de nuevos elementos "a fin de completar la obra médica con la social que, día a día, la realidad exige". ${ }^{12}$ Así justificó Dezeo en el discurso inaugural de la EVHS- UNLP la necesidad de formar a las mujeres:

Nadie más indicada que la mujer para esta función; pues en ella son innatos los sentimientos altruistas y el amor en

11. Pilades Dezeo, médico higienista, docente, con una amplia trayectoria en actividades extensionistas y, sobre todo, en la formación de la EVHS-UBA, fue el promotor para la creación de la EVHS-UNLP durante los años 1937 y 1938, y su director hasta el año 1942 en que murió. Su preocupación por la educación higiénica del "pueblo" y por la difusión de tales conocimientos lo empujó construir una Escuela para Visitadoras de Higiene Social en la Universidad Nacional de La Plata. Se desempeñó también como Jefe de la División de Higiene y Servicio Social de la Dirección de Maternidad e Infancia y como médico en los hospitales Alvear y Tornú.

12. DEZEO, Pilades, Discurso inaugural de la Escuela para Visitadoras de Higiene Social, Imprenta y Casa Editora Coni, Buenos Aires, 1938, p. 1. 
sus diversas manifestaciones; ella es el bálsamo y consuelo para todo dolor $\mathrm{y}$ es fuente inagotable de perseverancia, resignación y paciencia. Condiciones básicas, indispensables, para que la hagan insustituibles en tareas de sacrificio, como son las que debe cumplir todo trabajador social. $^{13}$

Los fundamentos sobre los que se estructuró la convocatoria a las mujeres parte de la asociación de la feminidad con el mundo de la domesticidad, y de la masculinidad con los roles directivos, establecidos en función de la diferencia sexual sobre la que se organiza la división patriarcal del trabajo, no solo en el hogar entre marido y esposa, sino también en los puestos de trabajo de la sociedad civil. ${ }^{14} \mathrm{Es}$ decir, que si bien la división sexual del trabajo establece espacios y tareas diferenciales para hombres y mujeres, las fronteras que separan lo público de lo privado resultan permeables a los intercambios entre sí aunque mantienen y reproducen las marcas de género. $\mathrm{La}$ confluencia entre patriarcado y capitalismo hacen de la mujer una suerte de "experta" en las tareas domésticas de asistencia, que no sólo son útiles a la unidad familiar, sino también, a la totalidad de la comunidad y al sistema capitalista, a pesar de ser desvalorizadas en su función productiva. ${ }^{15} \mathrm{La}$ infravaloración de las tareas domésticas facilita que el Estado especialice a las mujeres en aquellas tareas que pueden solaparse como "funciones naturales", pero, para la que necesitan, en el nuevo ordenamiento institucional, un título que las enaltezca como un agente específico

\section{3. Ídem, pp. 6-7.}

14. PATEMAN, Carol, El contrato sexual, Editorial Anthropos, Barcelona, 1995, pp. 9-17.

15. ARTOUS, Antoine, Los orígenes de la opresión de la mujer, Editorial Fontamara, Barcelona, 1982, p. 19.

98 y legitimado en el orden de género del capitalismo de principios del siglo XX.

Las características amorosas, altruistas, de resignación y perseverancia adjudicadas a las mujeres no fueron suficientes para la intervención sobre la cuestión social, de hecho las obras que habían realizado desde la beneficencia no podían auxiliar las necesidades de los nuevos desplazados luego de la crisis de 1930. Era necesaria una formación en salud pública, profilaxis, prevención social que permitiera intervenir sobre problemáticas sociosanitarias específicas; tal como señaló Dezeo:

Luchar con eficacia contra la intemperancia de las costumbres, contra la morbimortalidad materna e infantil, contra las enfermedades que desgastan al individuo, abrevian la vida o taran la descendencia, degenerando la raza. ${ }^{16}$

La EVHS establecía una formación de dos años, el primero de orden preparatorio $y$ común a todas, y el segundo dedicado a alguna de las especializaciones: Visitadora de Higiene Escolar, Visitadora para la Profilaxis de la Tuberculosis (estas dos especialidades también estaban en la EVHS - UBA) Visitadora en Puericultura, y por último Visitadora para Puericultura y Obstetricia. ${ }^{17}$ Puesto que la EVHS era

16. DEZEO, Pilades, Discurso..., Op. Cit., p. 9.

17. Cada una de ellas requería distintos antecedentes en su formación lo que demuestra que las visitadoras tenían un recorrido previo por las instituciones educativas, por ejemplo, las estudiantes que se especializaran en Profilaxis de Tuberculosis o Puericultura se les demandaba comprobar el cursado de estudios secundarios, normales o especiales incluso siendo parciales, y en caso de no ser así deberían rendir un examen de ingreso. A las interesadas 
arancelada y además requería de estudios previos para cada especialidad, podemos suponer que las estudiantes pertenecían a una clase media ascendente, que en su interés por participar como agentes del servicio social laico podían solventar los gastos de su formación profesional universitaria. Además, la posibilidad de iniciar una carrera como auxiliar de la medicina preventiva dentro de la universidad les posibilitó a estas mujeres la inserción en el proceso de transformación material e ideológica de los sectores populares, hecho que les suministró una cuota de poder, prestigio y distinción social.

Las labores que se esperaban de las visitadoras eran: trabajar junto al médico para la conservación o restablecimiento de la salud individual o colectiva, realizar propaganda para atraer al individuo o la familia a la institución preventiva o curativa, presentar los antecedentes mórbidos o económicos sociales de la situación que debían remediar, realizar el levantamiento de interrogatorios con una ficha clínico social, estudiar casos puntuales, gestionar la derivación a instituciones específicas, documentar las mismas y las soluciones aplicadas, colaborar con la acción del médico a través del asesoramiento "sobre las causas sociales que obstaculizan la salud o bienestar del niño", atender y explicar "las prescripciones dadas por el facultativo", dar educación sanitaria a las madres, ofrecer conferencias en clubes y escuelas para la

en el tratamiento de la Higiene Escolar se les pedía la presentación del título de maestra; mientras que a las dedicadas a la especialización en Obstetricia y Puericultura se les solicitaba ser parteras diplomadas de la UNLP o alumnas de tercer año de dicha escuela a condición de seguir el segundo año de la EVHS luego de finalizado el tercero como obstetras. Ver: Ordenanza de Creación de la Escuela para Visitadoras de Higiene Social, UNLP, La Plata, 1938. extensión de los preceptos de higiene, entre otras tareas.

Las últimas actividades descriptas fueron importantes ya que la preocupación por la "ignorancia" de los sectores populares hizo imprescindible la difusión educativa, a través de conferencias en clubes, en sociedades de socorros mutuos, en escuelas, hasta disertaciones radiales. Desde la radio las nuevas profesionales se colocaron en un espacio de difusión pública compartido con médicos higienistas que eran parte de la LPTPBA y con otros que eran parte de la FCM UNLP. Desde su participación radial pusieron en jaque los límites entre el espacio público y privado e invitaron a reconfigurar estos espacios de poder disertando no solo como auxiliares de los médicos, sino, como conocedoras y expertas del tratamiento sobre los males sociales y sanitarios de la población. Desde esa posición pudieron enunciar en voz propia sus saberes, e incluso persuadir con ellos a los sectores "ignorantes" y a los legistas, médicos y hombres de gobierno.

\section{El paisaje sonoro en la capital provincial y la intervención femenina}

La radio facilitó el proceso de integración cultural con el ingreso incuestionable en los hogares; sin necesidad de solicitar una visita o ingresar por algún control sanitario las voces se mezclaron en el paisaje sonoro doméstico. Entre las década de 1920 y 1940 la radio ocupó un lugar de privilegio entre los medios de comunicación masivos. Desde la primera transmisión experimental del país en 1920, Argentina estuvo entre los países de avanzada en la radiodifusión. Años más tarde, 
con la inclusión del parlante, las trasmisiones alcanzaron mayor audiencia y dos décadas después la radio pasó a ser un artefacto familiar en los hogares, incluso se estima que había una cada diez habitantes, lo cual indica que las trasmisiones tenían un alto alcance. ${ }^{18}$ La transmisión del turismo carretera y del radioteatro colaboró en la difusión de prácticas, costumbres e imaginarios que no estaban tan difundidos en las áreas más alejadas de las urbes.

LaPlata,caracterizada por emprenderacciones que condujeran las formas de convivencia, contó tempranamente con dos radios de amplio alcance nacional. En el año 1924 se creó la Radio Universidad LR11 reconocida por ser la primera radio universitaria del mundo y la segunda radio del país. ${ }^{19}$ Su tarea era convertirse en un elemento de extensión para la enseñanza, la investigación y la divulgación científica y cultural, hecho que la distinguió como promotora del conocimiento científico a través de la voz de agentes de la sociedad científica que tendieron puentes con la comunidad, y también entre docentes y alumnos. ${ }^{20}$ Estos fines la diferenciaron de otras radios surgidas en esos años cuyo objetivo era pertenecer al mundo comercial y del espectáculo.

Si bien comenzó con una pequeña antena que operaba de manera experimental y como herramienta de educación popular, para 1933 el entonces presidente de la UNLP, Ricardo

18. TORRE, Juan Carlos (director) Los años peronistas 1945-19455, colección Nueva Historia Argentina, T. VIII, Sudamericana, Buenos Aires, 2002, pp. 257- 311.

19. ANTONUCCI, Sergio, BENEITEZ, Maria Elena, GABAY, Sandra, TURCONI, Omar Enrique, Radio UNLP. 85 años. 1924- 2009, Edulp, La Plata, 2009.

20. Ídem, p. 19.
Levene, creó la Comisión de Transmisiones por Radiotelefonía a fin de ubicarla al servicio de un plan orgánico que facilitara la tarea de difusión y un año después inició un plan de inversiones para modernizar el aparato transmisor.

La Radio Universidad no fue la única de la región. En el año 1937, el gobernador conservador Manuel Fresco fundó Radio Provincia LS11 con el objetivo de establecer una herramienta de gestión. Hasta el momento no existía una radio oficial a nivel nacional, con la intervención del Ministro de Gobierno Roberto Noble (quien luego fundó el diario Clarín) se organizó en forma definitiva el servicio oficial de Radiodifusión por el decreto provincial No 51 en marzo de 1937 que constituyó a la Radio Provincia como el primer broadcasting oficial del Estado Argentino en dependencia de la Dirección de Correos y Telégrafos de la Nación. ${ }^{21}$

A través de la radiodifusión, el gobierno de Fresco realizó propaganda partidaria, reemitió programas de difusión nazi, es decir, utilizó los artilugios de la comunicación moderna para ubicar en un rol protagónico sus lemas de disciplinamiento social. Además de los objetivos de radiodifusión oficial, se pretendió generar contacto con los habitantes de la provincia para informarles de la gestión administrativa, contribuir al desarrollo de la cultura y brindar esparcimiento con un elevado criterio artístico alejado del

21. JARA, Juan Carlos, BOSARO, Leopoldo, LS 11 Radio Provincia. 60 años, Gobierno de la Provincia de Buenos Aires, Secretaria de Comunicación Social, La Plata, 1997, pp. 3. Vale aclarar que en el mes de julio del mismo año el Presidente Agustín P. Justo funda la Radio del Estado, actualmente Radio Nacional. 
lenguaje chabacano y arrabalero, que podía ser censurado. ${ }^{22}$ Esta radio, al igual que la dependiente de la UNLP, tampoco contaba con financiamiento comercial y por ello durante largos períodos no contrataba profesionales para su programación y esta se limitaba a la transmisión de música clásica.

Ambas radios tenían propuestas claramente diferenciadas. La Radio Universidad apuntó a la difusión de la cultura y el conocimiento universitario, y la otra, funcionó como herramienta de gestión de gobierno. Aún resta indagar sobre la relación entre la creación de Radio Provincia como parte de la oposición que existía entre Fresco y la Universidad, a la que consideraba un foco de subversión comunista plagado de indiferencia patriótica. Aún así, y dejando esta inquietud para otro análisis, cabe destacar que ambas emisoras se ocuparon de difundir conferencias realizadas por agentes del ámbito sanitario.

Durante los primeros años de radiodifusión en Argentina las mujeres tuvieron una participación que podríamos considerar desafiante. En línea con lo que sostiene Christine Ehrick consideramos que las mujeres que participaban en radio, sobre todo en el radioteatro, pudieron trastocar los ámbitos tradicionales de género y las demarcaciones entre espacios público y privado, por lo que la radio ayudó a la reformulación y renegociación de las relaciones entre los género, y además otorgó un lugar de encuentro entre las que hablaban y eran oídas. De esta manera, las mujeres podían combinar sus quehaceres domésticos y combatir el aislamiento característico de la época, invitando a las féminas a una

22. Ídem, pp. 6-8. comunidad ampliada de vida moderna y esfera pública. ${ }^{23}$

El hecho de intervenir el paisaje sonoro desde su voz como mujeres implicó la emergencia en el ámbito público de un sonido disonante que con antelación no cobraba semejante audibilidad. Esto, además, invitó a reconfigurar la relación entre público y privado, especialmente en un momento de expansión del Estado y de crecimiento del movimiento feminista en Argentina, donde las mujeres interrumpieron la univocidad del paisaje sonoro masculino. ${ }^{24}$ Las visitadoras se valieron de su lugar como difusoras de la educación sanitaria y trabajadoras de la prevención para irrumpir en el silencio a través de la enseñanza de conceptos de higiene como también de conocimientos producidos por sus experiencias a fin de persuadir al público y desestabilizar las jerarquías de género y profesionales.

\section{"La tuberculosis es una enfermedad evitable y curable"}

La Sta. Delia Gordon, visitadora de higiene escolar egresada de la EVHS- UNLP, tituló de esta forma su disertación del día 19/1/1940 en Radio Provincia. Su exposición formaba parte de las políticas de difusión llevadas adelante por la Comisión de Propaganda Higiénica y Educación Sanitaria Popular de la LPTPBA a cargo del Dr. Alberto Zambrosco que fue el subdirector del Preventorio de niños tuberculosos, médico agregado del consultorio para niños y subdirector ad-

23. EHRICK, Christine, Radio and gendered... Op. Cit., pp. 2-4.

24. Ídem, pp. 13-14. 
honorem de la EVHS-UNLP. ${ }^{25}$ Desde 1939 realizaron conferencias semanales con el objetivo de difundir estrategias de lucha contra la tuberculosis a través de disertaciones de médicos y visitadoras de higiene pertenecientes a la EVHS, y también invitados del ámbito sanitario no vinculados al ámbito universitario. ${ }^{26}$

La tuberculosis estaba cargada de significados que excedían lo patológico y la acercaban a una plaga social donde los sectores gobernantes encontraban la decadencia materialy espiritual de la sociedad. ${ }^{27}$ Esta enfermedad pulmonar propagada por el bacilo de Koch circulaba en todos los ambientes, especialmente, en aquellos poco ventilados $\mathrm{y}$ húmedos que le permitían reproducirse fácilmente. Los espacios más frecuentes eran instituciones de encierro, cárceles, fábricas e incluso las viviendas familiares, principalmente, aquellas de sectores populares dado el hacinamiento en que vivían por sus condiciones precarias de subsistencia. Desde fines del siglo XIX se sabía que esta enfermedad era transmisible y no hereditaria, por lo que el Estado y otras organizaciones sociales no gubernamentales, como la LPTPBA, buscaron difundir información acerca de cómo evitar la transmisión.

La insistencia en que la tuberculosis era una enfermedad evitable y curable fue uno de

25. A partir del año 1942 en que muere Pilades Dezeo Zambrosco será Director interino de la EVHS-UNLP.

26. Durante los años 1939 y 1940 se brindaron alrededor de ochenta radioconferencias que han sido transcritas en cuatro volúmenes publicados por la Comisión de propaganda higiénica y educación sanitaria popular de Liga Popular Contra la Tuberculosis de la Provincia de Buenos Aires en La Plata en el año1940.

27. ARMUS, Diego, La ciudad impura..., Op. Cit., p. 16. 102 los enunciados desde el que se posicionaron las visitadoras para desterrar los temores de herencia de la enfermedad, lograr la atención de sus oyentes y alertar sobre la necesidad de iniciar acciones cotidianas y cambios de hábitos que cuidaran al conjunto de la población. Especialmente, apuntaron a los sectores populares. Por ejemplo la visitadora Elida Sciani en su conferencia "Concepto general de lucha antituberculosa. Recursos económicos y profilaxis antituberculosa" sostuvo:

Que por ignorancia, dejadez o miseria la mas de las veces, es donde el mal encuentra el cultivo, razón por la cual se debe provocar la intervención de los poderes públicos en la lucha contra la tuberculosis. ${ }^{28}$

La idea de asociar la ignorancia a los sectores populares se trasladó a los oyentes, a quienes, en cada una de las disertaciones, se volvía a explicar la historia de la tuberculosis y como repercutía en su salud. Por ello, en su disertación "La tuberculosis es una enfermedad evitable y curable", Delia Gordon detalló:

E1 mi- cro- bio: micro que quiere decir pequeño, bio: que quiere decir vida. Tan pequeño que su tamaño es de dos o tres milésimas, no obstante para ser tan pequeñito sabe seleccionar sus víctimas. ${ }^{29}$

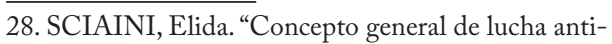
tuberculosa. Recursos económicos y profilaxis antituberculosa" (Conferencia No 46 26/2/1940), en Comisión de propaganda bigiénica y educación sanitaria popular, Liga Popular Contra la Tuberculosis de la Provincia de Buenos Aires, La Plata, 1940, p. 360.

29. GORDON, Delia, "La tuberculosis es una enfermedad curable y evitable" (Conferencia no 45 19/ 02/1940), en Comisión de propaganda higiénica y educación sanitaria popular, Liga Popular Contra la Tuberculosis de la Provincia de Buenos Aires, La Plata, 1940, p. 354. 
Sus discursos tuvieron un carácter propedéutico, cargados de explicaciones minuciosas que intentaban concientizar a los oyentes acerca del contagio de la enfermedad. Existió una pretensión de persuadir al público a través de los detalles con que argumentaron las problemáticas presentadas, que de alguna manera intentaban generar temor respecto de este microbio "tan pequeñito".

Desde su rol educativo como conferencistas las visitadoras se ocuparon de persuadir al público a través de dos mecanismos. En principio, como ya hemos dicho, con la explicación reiterada y pormenorizada de la enfermedad que se presentaba como un peligro inminente pero evitable en cada conferencia, y, por otro lado, a través de la descripción exhaustiva del trabajo que realizaban ellas y los médicos dentro de los dispensarios para mostrar la conveniencia de acercarse y evitar "males mayores". Así relató su experiencia como visitadora honoraria del Dispensario Central "Susana Araoz Alfaro" Nélida Tebaldi en la conferencia titulada "Como trabaja un dispensario de lucha antituberculosa”:

Si se trata de un adulto que por primera vez concurre al Dispensario pasa al Consultorio de Adultos donde es recibido por la Visitadora quién inicia su función por un interrogatorio. Este interrogatorio le permitirá llenar una ficha llamada Primer Examen de Adulto: que consta de varias preguntas que se refieren a los datos de identidad personal: nombre, apellido, nacionalidad, profesión, etc... Luego le interroga sobre los antecedentes familiares. ${ }^{30}$

30. TEBALDI, Nélida, "Cómo trabaja un dispensario de lucha antituberculosa” (Conferencia No 50, 25/03/1940), en Comisión de propaganda higiénica y educación sanitaria
Con este interrogatorio querían saber si había personas posiblemente infectadas en la familia o allegados, por ello las preguntas sanitarias se extendían sobre aquellas personas con las que tenía vida íntima, sobre todo en inquilinatos y pensiones. Mediante esta presentación detallada, Tebaldi intentó generar cierta confianza persuadiendo al público acerca de la "simplicidad" que suponía acercarse al dispensario.

Es interesante que la labor de las visitadoras en tanto conferencistas y propagadoras de nuevos hábitos higiénicos (por ejemplo se mencionan los modos en que debe trapearse el suelo para evitar que se levante polvillo y con el los bacilos de Koch, o cómo debe hervirse la leche para evitar el contagio de tisis por la vía intestinal) no se limitó solo a persuadir sobre la inminencia de modificar sus costumbres, sino que también intentó promover la confesión de las prácticas cotidianas (si escupe, cómo lo hace, cuantas veces tose, si abre la ventana por la mañana, con quienes vive, entre otros) a través del acercamiento al dispensario y en los domicilios particulares, donde se relevaban datos sobre el nivel educacional del enfermo, la conducta moral, condiciones higiénicas de la casa y sus recursos económicos por medio de la Encuesta Social. En la detallada y extensa exposición de Tebaldi en la conferencia ya citada, explicitó cual era uno de los objetivos de las visitas domiciliarias:

La visitadora debe tratar entonces de que concurran al Dispensario no solo el sospechoso, sino todas las personas que habitan la casa; venciendo las resistencias

popular, Liga Popular Contra la Tuberculosis de la Provincia de Buenos Aires, La Plata, 1940, pp. 395- 396. 
que se le opongan por medio de la persuasión. ${ }^{31}$

La estrategia persuasiva apareció constantemente en las exposiciones de las visitadoras. Con ella intentaron convencer y familiarizar a la población sobre la importancia de acercarse al dispensario dada la simplicidad del tratamiento y conveniencia colectiva de hacerlo. Es destacable el hecho de que las visitadoras hayan reconocido la persuasión como un modo de intervención en las visitas domiciliarias que les posibilitó lograr con éxito su labor. Esto nos permite considerar que las acciones de estas mujeres no se basaron exclusivamente en un vínculo afectivo y amoroso, sino que eran conscientes de su capacidad argumental y racional para convencer a la población de seguir sus propuestas de cuidados sociosanitarios.

\section{"No es solo un problema médico, es una cuestión social"}

Las visitadoras sabían que sus acciones médicas profilácticas no bastaban para el mejoramiento de las condiciones de vida de la población, por ello la radio operó como una tribuna desde la cual llamar la atención de quienes en principio no era el público destinatario. La visitadora Elida Sciaini en su disertación "Concepto general de la lucha antituberculosa. Recursos económicos y profilaxis" destacó:

Para preservar la infancia se debe procurar la buena alimentación general y la vivienda higiénica para todos, no solo debemos completar y ampliar la

31. Ídem, p. 400. obra social de protección a la madre y al niño... deben generarse espacios de profilaxis indirecta, como leyes de trabajo, maternidades, cantinas maternales, casas aireadas, escuelas con inspección médica permanente, educación física adecuada, escuelas al aire libre. ${ }^{32}$

Reparemos entonces, ¿con quién dialoga Sciaini en su conferencia? ¿Es al público "ignorante" de los sectores populares a quien exige ampliar la obra social, dar leyes de trabajo y garantizar escuelas al aire libre? Evidentemente no. Parece que haciendo uso del privilegio de tomar la voz en público y durante los minutos que duró su conferencia se permitió alentar a la reflexión de los hombres de política y de leyes que tuvieran a su alcance estas modificaciones de orden sanitario y habitacional.

Desde las radioconferencias las visitadoras se colocaron en un umbral que trastocó el espacio público y privado, reconfiguraron su posición "privada" y auxiliar a través de la interrupción en un paisaje que era mudo para las mujeres. Ejemplo de ello es la disertación "La tuberculosis, como se adquiere y cómo se evita” de Sofía Ricci, quién encendió el espacio radial de este modo:

Cada media hora muere un tuberculoso. Esta verdad debe preocuparnos a todos, al pueblo y a sus dirigentes... El problema no puede ser exclusivamente médico, que es en gran parte una cuestión social. Es por ello que el día que se procure a las masas humanas una buena alimentación, una mejor vivienda y el conocimiento de una serie de medidas higiénicas se habrá dado

32. SCIAINI, Elida, "Concepto general de lucha antituberculosa..., Op. Cit., p. 365. 
un gran paso hacia el exterminio de este terrible mal. ${ }^{33}$

Este llamamiento a los dirigentes del país generó otra disonancia en el paisaje sonoro. Era una mujer exigiendo respuestas y soluciones para una enfermedad cuya solución no se limitaba a la intervención del médico, ni a las acciones que pudiesen tomar individualmente las familias de los sectores populares. Ricci extendió sus demandas la clase dirigente, y con ello intentó acortar las distancias entre el espacio político masculinizado y el espacio de cuidados en el que se ubicaba a las mujeres.

Sus voces invadieron el espacio radial con la autoridad de la experiencia como agentes del servicio social, con conocimientos sobre los tratamientos que se realizaban sobre las personas infectadas, sobre la alimentación de los niños, sobre las causas sociales de las enfermedades e incluso sobre como operaba cada instancia del dispensario. Con la descripción de sus acciones pretendían persuadir a los distintos públicos a los que indicaron insistentemente sobre la necesidad de realizar modificaciones en sus conductas, e incluso en sus estrategias políticas. En la enunciación de sus conocimientos y experiencias laborales podemos encontrar una búsqueda por la legitimación social de la nueva profesión que más allá del rol auxiliar de los médicos, pudieron delinear saberes y prácticas específicas por lo que exigían audibilidad y atención. ${ }^{34} \mathrm{La}$ estudiante de

33. RICCI, Sofia, "La tuberculosis, como se adquiere y cómo se evita", Ricci (Conferencia N 80 21/ 10/1940) en Comisión de propaganda higiénica y educación sanitaria popular, Liga Popular Contra la Tuberculosis de la Provincia de Buenos Aires, La Plata, 1940, pp. 638- 639.

34. Carlos Montaño indica que existen dos posturas diferentes acerca de la legitimidad en el trabajo social, una de
Hortensia Moretti en su conferencia titulada "Ahorrar a costa de la salud es comprometer el porvenir biológico" lo planteó del siguiente modo:

Yo, como todas mis compañeras, puestas al servicio de la Nación para salvaguardar las vidas de nuestros semejantes, pedimos se escuchen y valoren todo lo que se dice y hace en vuestro favor, para ver coronados nuestros esfuerzos de ser útiles y de conseguir así, un pueblo sano y hacer una Nación floreciente. ${ }^{35}$

La joven estudiante irrumpió en la sonoridad radial para aclarar que sus acciones debían ser valoradas, requerían de un respeto e importancia por la población puesto que sus intervenciones y consejos hacían a la nación misma. El pedido de escucha demuestra que el acto de hablar en la radio no garantizaba ser oídas y por ello se atrevió a exigirlo desde la radio justificando su utilidad para el bienestar de la comunidad.

\section{"Democratización de la cultura, indispensable para el éxito de la medicina"}

E1 Dr Alberto Zambrosco sintetizó con esta frase, los intereses de las radioconferencias

orden funcional, caracterizada por la demanda que ejerce el Estado en consonancia con las necesidades de los sectores hegemónicos por garantizar su dominio; y otra de carácter social, que proviene del vínculo establecido entre la trabajadora social y el sujeto usuario al que se dirigen sus prácticas. Ver: MONTAÑO, Carlos, La naturaleza del Servicio Social. Un ensayo sobre su génesis, su especificidad, su reproducción, Cortez Editora, San Pablo, 1998.

35. MORETTI, Hortensia, "Ahorrar a costa de la salud es comprometer el porvenir biológico" (Conferencia no 64 1/7/1940) en Comisión de propaganda higiénica y educación sanitaria popular, Liga Popular Contra la Tuberculosis de la Provincia de Buenos Aires, La Plata, 1940, p. 505. 
tituladas "Educación Sanitaria Popular y Propaganda Higiénica" ${ }^{36}$ realizadas desde la cátedra de Higiene Médica y Preventiva en cooperación con la EVHS durante el año 1942. ${ }^{37}$ En este ciclo de radioconferencias las temáticas a tratar no fueron solo enfermedades como la tuberculosis, se incluían los problemas de la maternidad e infancia, la asistencia social, la relación entre higiene y medicina social, donde médicos y visitadoras dieron cuenta de distintos abordajes. Hubo otras tres áreas de conferencias donde solo disertaron hombres: industrialismo, aspectos médicos legales y medicina preventiva.

En el primer grupo de conferencias "Problemas de maternidad e infancia" nos encontramos nuevamente con Nélida Tebaldi, quien dos años después de las conferencias brindadas desde la LPTPBA ocupó el cargo de jefa de clases prácticas de la materia Servicio Social en la EVHS. En esta ocasión, manifestó su preocupación acerca de las mujeres embarazadas que morían por su "incapacidad para sobrellevar la tremenda responsabilidad que su nuevo estado significa”. ${ }^{38}$ Esta reflexión se vincula con la

36. Educación Sanitaria y propaganda higiénica. Conferencias radiotelefónicas. Facultad de Ciencias Médicas, Cátedra de Higiene Médica y Preventiva, La Plata UNLP, 1942.

37. Si bien las memorias de la EVHS UNLP dan cuenta de la temprana participación en el programa de extensión universitaria a través de Radio Universidad, el primer libro editado de las conferencias es al que referimos en este apartado. Allí se destaca la participación de Visitadoras egresadas y estudiantes de la EVHS, como también estudiantes avanzados y médicos de la FCM- UNLP-

38. TEBALDI, Nélida, "El amparo de la mujer en su condición de madre o de futura madre", en Educación Sanitaria y propaganda higiénica. Conferencias radiotelefónicas. Facultad de Ciencias Médicas. Cátedra de Higiene Médica y Preventiva, UNLP, La Plata, 1942, pp. 23- 32. 106 preocupación por el binomio madre-hijo y el cuidado sobre las madres como parte de una cruzada por el futuro de la "raza" y, con ello, del progreso material y social de la Nación. ${ }^{39}$ En esta conferencia desarrolló un análisis procesual acerca del tratamiento que debía tener una mujer embarazada, inicialmente con el seguimiento eugenésico de los padres previo a la gestación, luego con la descripción de los estudios generales en los consultorios obstétricos, para finalmente, luego del parto, presentar las utilidades de las maternidades con el fin de persuadir a las futuras madres sobre los beneficios de acercarse al centro de atención socio sanitario para un mayor cuidado e instrucción.

Las visitadoras en Radio Universidad disertaron como conocedoras de las problemáticas sobre las madres e hijos $\mathrm{y}$, con esta posición de autoridad desde el saber de su experiencia, generaron una operación por la cual nuevamente una vivencia de orden privado era presentada al espacio público como una problemática general y no individual. Así, la visitadora Tebaldi cuestionó la problemática de los hijos ilegítimos y la responsabilidad de los padres y del Estado por asistir a esas madres, e incluso se atrevió a exigir que se modifique la legislación que calificaba a los hijos como ilegítimos sin intervenir sobre las responsabilidades paternas, por ello sentenció "que se reconozca por la ley la maternidad de hecho y se disponga de una investigación de la paternidad". ${ }^{40}$

39. BIERNAT, Carolina y Karina RAMACCIOTTI, Crecer y Multiplicarse. La politica sanitaria materna infantil. Argentina, 1900-1960, Buenos Aires, Biblos, 2013, p. 103.

40. TEBALDI, Nelida, "El amparo de la mujer...", Op. Cit., p. 29. 
Al considerar la maternidad e infancia no como situaciones individuales y de orden exclusivamente doméstico, sino también como problemas sociales que concernían a las mujeres en general, como también a las autoridades sanitarias, las visitadoras pretendieron hacer llegar sus propuestas a estos sectores a través de la radio, y además tender complicidades con la audiencia femenina. En este sentido, Alfina Copani, visitadora de higiene social y secretaria de la EVHS, planteó hipotéticas soluciones a la mortalidad materna en su disertación "La mortalidad materna y su profilaxis" como la siguiente:

Es necesario para ello crear una organización de asistencia y previsión médico-social que beneficie a todas las madres de la República, y abogar por la implantación de medidas de mejoramiento de nivel económico, educativo, higiénico y moral de la población. ${ }^{41}$

Nuevamente, las visitadoras dialogaron no solo con el público "ignorante", sino también con el conjunto de universitarios que eran parte del auditorio de la Radio UNLP. Tal vez por ello, a diferencia de las conferencias en Radio Provincia, en esta emisora la estrategia persuasiva se valió de argumentos del discurso sanitario, jurídico y sociológico que estuvo cargado de estadísticas nacionales sobre los niveles de mortalidad, referencias a leyes y ordenanzas para la protección del binomio madre hijo. ${ }^{42}$ Las visitadoras se presentaron

41. COPANI, Alfina, "La mortalidad materna y su profilaxis", en Educación Sanitaria y propaganda higiénica. Conferencias radiotelefónicas, Facultad de Ciencias Médicas. Cátedra de Higiene Médica y Preventiva. UNLP, La Plata, 1942, pp. 41-50

42. Algunas de las leyes que aparecen mencionadas en las radioconferencias son: Ley 11.933 del año 1934 que así en el paisaje sonoro universitario como profesionales de la problemática, capacitadas para debatir e incluso proponer soluciones. Su voz en el éter hizo audible y visibilizó una problemática muy sensible entre la comunidad científica.

Las experiencias como agentes del servicio social habilitaron la voz de las visitadoras como expertas en sus áreas de intervención específicas. En el ciclo destinado a la Asistencia Social, María Haydee Aguilar, visitadora egresada de la EVHS- UNLP y visitadora del Instituto de Maternidad y Asistencia Social del Hospital Parmenio Piñero en Capital Federal, compartió la preocupación por dos de los problemas desprendidos de su intervención en las maternidades: la ilegitimidad y las carencias económicas de las madres. Para dar cuenta de esta situación sustentó su análisis con la presentación detallada de estadísticas realizadas por ella en Capital Federal y sobre el final de su exposición titulada "Importancia del servicio social en la maternidad” Aguilar destacó:

al hacer estos enunciados, queremos dejar bien aclarados que, con ellos, no pretendemos realizar, en las salas de Maternidad, la obra integral que requiere la solución de sus múltiples problemas, por cuanto una obra educativa es imposible de realizar durante el breve tiempo que estas mujeres permanecen internadas en dichos

prohíbe el trabajo de las mujeres un mes antes y 45 días después del alumbramiento, la ordenanza No 7915 del año 1936 por la que los servicios de maternidades de los hospitales municipales se elevaron a la categoría de Institutos de Maternidad y Asistencia Social. Se incluye también la Ley 12.331 de profilaxis antivenérea como parte del aspecto preventivo y eugenésico para el mejoramiento de la salud maternal, y por supuesto la ley 12.341 de Protección a la Maternidad e Infancia. 
servicios, lo que solo podrá realizarse en Hogares Refugio... ${ }^{43}$

¿A quién le hablaba Aguilar con estas palabras? ¿Se expidió de no poder resolver el malestar económico y el abandono de las mujeres que se acercaban a las maternidades o en realidad hizo un llamamiento a los sectores gobernantes para que intervinieran y complejizaran la atención sobre las mujeres madres? Claramente extendió una exigencia a los sectores gobernantes que tenían la posibilidad de ejecutar una obra integral para el bienestar de las mujeres madres. Esta posición de defensa a los intereses de la maternidad junto con la exigencia de nuevos servicios interrumpió el silencio de las mujeres.

\section{Consideraciones finales}

La difusión y educación sanitaria formó parte de las acciones que llevaron adelante las visitadoras de higiene. Como hemos visto, se esperaba que hicieran gala de sus dotes femeninas de resignación, perseverancia y sacrificio para que en consonancia con los deberes maternales atribuidos a las mujeres de sectores medios en profesiones sociosanitarias, estimularan cambios de hábitos en la vida cotidiana de los sectores más vulnerables. Sin embargo, su acción superó los límites de intervención doméstica, y a través de las radios platenses alcanzaron una mayor difusión de sus preocupaciones y propuestas, en las que desarrollaron

43. AGUILAR, María Haydee, "Importancia del servicio social en la maternidad", en Educación Sanitaria y propaganda higiénica. Conferencias radiotelefónicas, Facultad de Ciencias Médicas, Cátedra de Higiene Médica y Preventiva, UNLP, La Plata, 1942, pp. 125-126.

108 argumentos persuasivos basados en su experiencia en el ámbito sociosanitario.

La intervención radial facilitó el proceso de integración de las mujeres en el espacio sonoro, donde sus reflexiones y aportes se hicieron audibles a la población y no solo a los sectores con quienes trabajaban; además el hecho de que las conferencias coincidieran con los primeros años del desarrollo de la EVHS- UNLP les habría posibilitado cierta legitimación social de su profesión.

En función de las conferencias presentadas podemos delinear algunas diferencias. Las conferencias de la LPTPBA se dieron en consonancia con los objetivos de disciplinamiento social que tuvo Radio Provincia desde su fundación; las disertaciones de las visitadoras manifestaron una preocupación específica sobre los sectores populares y la difusión de las estrategias preventivas para la modificación de destinados a evitar el contagio de la tuberculosis. También intentaron persuadir al público para generar su acercamiento al Dispensario donde además de prevenir la enfermedad obtendrían información sobre la vida privada de estos sujetos. Por su parte, en Radio Universidad las conferencias integraron las tareas de extensión universitaria, por ello el uso de argumentos, leyes, estadísticas, e incluso, de las investigaciones de las visitadoras parecen evidenciar que buscaron como interlocutores a la comunidad científica y gobernante, sobre quienes extendieron sus pretensiones persuasivas.

Resulta destacable que aún privadas de derechos de ciudadanía política, las visitadoras se presentaron en el espacio radial 
como partícipes activas de la comunidad. A través de su intervención en el paisaje sonoro construyeron complicidades con otras mujeres por medio de la presentación de problemas privados (como la alimentación, el cuidado de los niños, la higiene, entre otros) que se presentaban como problemas públicos. Con esta operación las visitadoras acortaron las distancias entre las experiencias individuales de las mujeres madres para demostrar que muchos de sus padeceres no eran un problema individual, sino un problema estructural sobre el que el Estado y sus legisladores debían intervenir.

En este sentido, las disertaciones radiales de las visitadoras de higiene social en la ciudad de La Plata nos permiten ponderar acciones creativas, persuasivas, argumentales, de producción académica por medio de las que desafiaron el lugar exclusivamente amoroso y conservador con el que han sido consideradas. La confianza en sus conocimientos y experiencias les permitieron contar con argumentos sólidos para intentar convencer a la población de acercarse a las instituciones sanitarias, y también de realizar modificaciones en sus conductas, e incluso, en sus estrategias políticas.

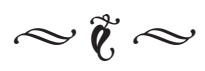

Recibido: 25-06-2016

Aceptado: 25-10-2016

Publicado: 30-12-2016 


\section{Bibliografía}

ANTONUCCI, Sergio, BENEITEZ, Maria Elena, GABAY, Sandra, TURCONI, Omar Enrique, Radio UNLP. 85 años. 1924- 2009, Edulp, La Plata, 2009.

ARMUS, Diego, La ciudad impura. Salud, tuberculosis y cultura en Buenos Aires, 1870-1950, Edhasa, Buenos Aires, 2007.

ARRUA, Néstor, "Médicos higienistas y visitadoras frente a la tuberculosis en la ciudad de La Plata (19351943)", Revista Cátedra Paralela, Rosario, 2014, No 11.

ARTOUS, Antoine, Los orígenes de la opresión de la mujer, Editorial Fontamara, Barcelona, 1982.

BIERNAT, Carolina y Karina RAMACCIOTTI, Crecer y Multiplicarse. La politica sanitaria materno infantil. Argentina, 1900-1960, Biblos, Buenos Aires, 2013.

DI LISCIA, Maria Silvia, "Renovación de la historia sobre instituciones, profesionales y salud" en BIERNAT, Carolina, RAMACCIOTTI, Karina (editoras) Historia de la salud y la enfermedad. Bajo la lupa de las ciencias sociales, Biblos, Buenos Aires, 2014, pp117- 129.

EHRICK, Christine, Radio and gendered soundscape. Women and broadcasting in Argentina and Uruguay, 1930- 1950,Cambridge University Press, Cambridge, 2015.

GRASSI, Estela, La mujer y la profesión de asistente social: el control de la vida cotidiana, Humanitas, Buenos Aires, 1989.

GONZALEZ LEANDRI, Ricardo, "Madurez y poder. Médicos e instituciones sanitarias en la Argentina a fines del siglo XIX”, en Entrepasados, Buenos Aires, 2005, № 27, pp. 133- 150.

GRAU, Carlos, La Sanidad en las ciudades y pueblos de la provincia de Buenos Aires, Dirección de Impresiones Oficiales, La Plata, 1954.

JARA, Juan Carlos, BOSARO, Leopoldo, LS 11 Radio Provincia. 60 años, Gobierno de la Provincia de Buenos Aires, Secretaria de Comunicación Social, La Plata, 1997.

LOBATO, Mirta, Historia de las trabajadoras en la Argentina (1869-1969), Edhasa, Buenos Aires, 2007.

MARTIN, Ana Laura, "Mujeres y enfermería. Un asociación temprana y estable, 1886- 1940" en BIERNAT, Carolina, CERDÁ; Juan Manuel; RAMACCIOTTI, Karina (directores) La salud pública y la enfermería en Argentina, Universidad Nacional de Quilmes, Bernal, 2015, pp. 257-286.

MONTAÑO, Carlos, La naturaleza del Servicio Social. Un ensayo sobre su génesis, su especificidad, su reproducción, Cortez Editora, San Pablo, 1998.

NARI, Marcela, Politicas de Maternidad y Maternalismo Politico, Biblos, Buenos Aires, 2004.

OLIVA, Andrea, Trabajo social y lucha de clases, Imago mundi, Buenos Aires, 2007. 
PARRA, Gustavo, Antimodernidad y Trabajo Social. Orígenes y expansión del Trabajo Social Argentino, Espacio Editorial, Buenos Aires, 2007.

PATEMAN, Carol, El contrato sexual, Editorial Anthropos, Barcelona,1995.

RAMACCIOTTI, Karina y VALOBRA, Adriana, "Modernas esculapios: acción política e inserción profesional”, en LIZETTE, Jacinto y SCARZANELLA, Eugenia. Género y Ciencia en América Latina: mujeres en la academia y en la clinica, Editorial Iberoamericana, Madrid, 2011, pp. 23-51.

REITANO, Emir. Manuel Fresco. Entre la renovación y el fraude, Archivo Histórico de la Provincia de Buenos Aires, La Plata, 2005.

TORRE, Juan Carlos y PASTORIZA, Elisa, "La democracia del bienestar" en TORRE, Juan Carlos Los años peronistas 1943-1955, Colección Nueva Historia Argentina, T. VIII, Sudamericana, Buenos Aires, 2002, pp. 257-311.

VALLEJO, Gustavo, Escenarios de la cultura cientifica argentina. Ciudad y Universidad (1882-1955), CSIC, Madrid, 2007. 